\section{Eltern sparen oft mit Schmerzmitteln} Über 80\% der pädiatrischen Operationen in den USA werden ambulant
durchgeführt. Die postoperative Schmerzbehandlung liegt dann vorwiegend
in der Hand der Eltern. Dies dient jedoch nicht immer dem Wohl der Kinder.

D ie Behandlung postoperativer Schmerzen bei Kindern in der häuslichen Umgebung war Ziel einer US-amerikanischen Untersuchung. Dazu fragten die Autoren 132 Eltern vor der Operation ihres Kindes nach ihrer allgemeinen Einschätzung von Schmerzen bei Kindern und nach ihrer Meinung zu Schmerzmitteln. Bei Entlassung der Kinder bekamen sie dann genaue Anweisungen zur Schmerztherapie und den

Auftrag, ein Schmerztagebuch für ihre Kinder zu führen. Bei den verschriebenen Medikamenten handelte es sich, je nach Operation und Arzt, um Paracetamol, Paracetamol mit Kodein oder Ibuprofen.

Das Ergebnis war deutlich - es besteht jede Menge Aufklärungsbedarf. Zum Beispiel waren 36\% der Eltern der Meinung, dass Kinder ihre Schmerzen immer durch Schreien oder Weinen äußern,

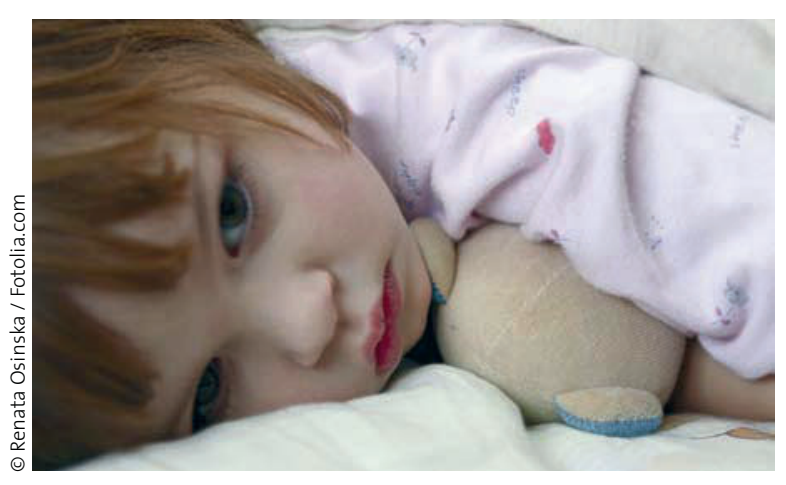

Nicht alle Kinder zeigen ihren Schmerz durch Weinen oder Schreien.

\section{Hygiene-Hypothese mit umgekehrtem Vorzeichen}

Der frühe Kontakt mit Mikroorganismen soll eine protektive Wirkung gegen allergische Erkrankungen haben. Eine deutsche Analyse weist jedoch darauf hin, dass bei Vorliegen einer Filaggrin-Mutation genau das Gegenteil der Fall sein kann.

E in deutsches Autorenteam wertete - Daten der beiden Kohortenstudien LISAplus und GINIplus aus. Analysiert wurden die Kinder, bei denen eine Testung auf Filaggrin-Mutationen durchgeführt worden war $(\mathrm{n}=2.867)$. Bei 6,7\% (LISAplus) und 6,0\% (GINIplus) waren diese Mutationen nachweisbar. Mit der Auswertung sollte geklärt werden, ob die Anwesenheit älterer Geschwister (und der damit postulierte vermehrte Kontakt mit Keimen) einen protektiven Effekt auf die Entwicklung von Ekzemen zeigt.
Ältere Geschwister hatten nach diesen Daten keinen signifikanten Einfluss auf die Entwicklung von Ekzemen: Die entsprechende Odds Ratio (OR) betrug bei den Teilnehmern der LISAplus-Studie 1,21 (95\%-Konfidenzintervall, CI: 0,93-1,58). Für Studienteilnehmer, die keine Kindertagesstätten besuchten, lag das Risiko bei 1,01 (CI: 0,74-1,37). In der GINIplusStudie ergaben sich Werte von 1,00 (CI: 0,81-1,24) bzw. 1,00 (CI: 0,80-1,24).

Bei gleichzeitigem Vorliegen einer Filaggrin-Mutation und der Anwesenheit älterer Geschwister lag das Ekzemrisiko in
$30 \%$ dachten, dass Kinder den Eltern immer sagen, wenn sie Schmerzen haben. In Schmerzmitteln sahen viele Eltern eine Gefahr: 73\% fürchteten sich vor möglichen Nebenwirkungen. Über die Hälfte aller Eltern waren der Meinung, das verschriebene Schmerzmittel mache die Kinder abhängig. 37\% dachten, ein Analgetikum wirke umso besser, je sparsamer es verwendet werde. Je mehr Bedenken die Eltern bezüglich der Schmerzmittel hatten, desto weniger davon gaben sie ihren Kindern. Selbst bei einem medianen Wert von sechs auf der Schmerzskala, der signifikante Schmerzen anzeigte, bekamen die Kinder im Mittel nur eine Dosis eines Analgetikums.

Fazit: Angesichts der Tatsache, dass viele Kinder nach einer Operation Schmerzen haben und deren Behandlung in den meisten Fällen den Eltern überlassen ist, sollten diese besser über Schmerzmedikamente, ihre Nebenwirkungen und ihren postoperativen Einsatz informiert werden, schlussfolgern die Autoren. ib

Rony RY et al. Parental postoperative pain management: attitudes, assessment, and management. Pediatrics 2010; 125: e1372-8

der LISAplus-Studie bei 1,51 (CI: 0,623,68, keine Signifikanz). In der GINIplusStudie zeigte sich hier ein signifikant erhöhtes Risiko von 2,38 (CI: 1,09-5,17). Bei den Kindern, die keine Kindertagesstätten besuchten, war in beiden Studien eine signifikante Zunahme des Ekzemrisikos nachweisbar (OR: 3,27, CI: 1,149,36 bzw. OR: 2,41, CI: 1,06-5,48).

Fazit: In dieser Analyse konnte der in früheren Studien gezeigte protektive Effekt von älteren Geschwistern auf das Ekzemrisiko nicht bestätigt werden. Bei einer nachgewiesenen Filaggrin-Mutation erhöhte die Anwesenheit von Geschwistern sogar das Risiko.

Cramer $\mathrm{C}$ et al. Elder siblings enhance the effect of filaggrin mutations on childhood eczema: Results form the 2 birth cohort studies LISAplus and GINIplus. J Allergy Clin Immunol 2010; 125: 1254-60 